\title{
Anti-Neutrophil Cytoplasmic Antibody- Associated Vasculitis, Large Vessel Vasculitis and Kawasaki Disease in Japan
}

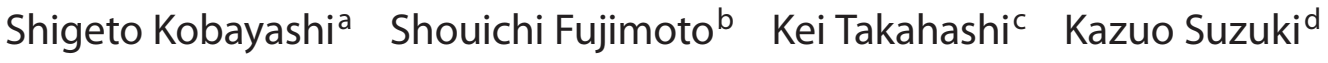 \\ aRheumatology, Juntendo Koshigaya Hospital, Saitama, b Dialysis Division, University of Miyazaki Hospital, \\ Miyazaki, 'Department of Pathology, Toho University Ohashi Medical Center, Tokyo, and d Inflammation Program, \\ Department of Immunology, Chiba University Graduate School of Medicine, Chiba, Japan
}

\section{Key Words}

Myeloperoxidase $\cdot$ Anti-neutrophil cytoplasmic antibody •

Vasculitis • Epitope analysis · Rapidly progressive

glomerulonephritis $\cdot$ Microscopic polyangiitis •

Kawasaki disease $\cdot$ Systemic vasculitis $\cdot$ Epitopes

\begin{abstract}
Based on studies comparing the prevalence of anti-neutrophil cytoplasmic antibody (ANCA)-associated vasculitis (AAV) between Japan and Europe, we have learned that the difference may be due to genetic background and environmental factors, but not to diagnosis or ELISA system for myeloperoxidase and proteinase-3 ANCA. In Japan, microscopic polyangiitis is the most common among AAV, but Wegener's granulomatosis was present in less than 2 per million patients. Also, one study from Hokkaido reported only 16 patients in a 27-year time frame. A recent retrospective study of renal vasculitis between 2000 and 2004 from Miyazaki prefecture in Japan reported an incidence of microscopic polyangiitis of 14.8 per million, but no patients with Wegener's granulomatosis or Churg-Strauss syndrome. In the present review, we focus on ANCA-related vasculitis in Japan: (1) AAV and large vessel vasculitis - Takayasu's arteritis and giant cell arteritis; (2) primary renal vasculitis; (3) epitopes of myeloperoxidase-ANCA in vasculitis in the Japa-
\end{abstract}

nese population and comparison of ANCA-ELISA systems in Japan and Europe, and finally (4) children with vasculitis in Japan involving Kawasaki disease - a systemic vasculitis.

Copyright $\odot 2010$ S. Karger AG, Basel

\section{Introduction}

In the late 1990s, discussions suggested more widespread variations in disease frequency, with Wegener's granulomatosis (WG) being rare in Japan, where proteinase-3 (PR3) anti-neutrophil cytoplasmic antibody (ANCA) antibodies were rarely detected. The majority of patients with renal vasculitis have myeloperoxidase (MPO) antibodies and are classified as having microscopic polyangiitis (MPA). Furthermore, prevalence studies from Japan have estimated that WG is present in less than 2 per million patients, suggesting an incidence of less than 0.1 million per year, according to the $\mathrm{Re}$ search Group of Epidemiology and Intractable Diseases, supported by a grant of The Ministry of Health, Labour and Welfare, Japan $[1,2]$.

The hypothesis that the different genetic and environmental background between Japan and Europe would lead to a difference in the clinical phenotype has been investigated. One of the original hypotheses that the distri-

\section{KARGER}

Fax +41613061234 E-Mail karger@karger.ch www.karger.com

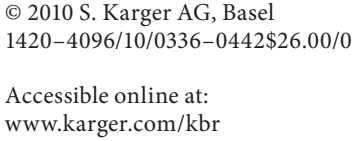

Inflammation Program, Department of Immunology

Chiba University Graduate School of Medicine

Inohana 1-8-1, Chuo-ku, Chiba 260-8670 (Japan)

Tel. +81 43221 0831, Fax +81 43221 0832,E-Mail ksuzuki@faculty.chiba-u.jp 
bution of vasculitis phenotypes and ANCA specificities would vary with latitude has not been confirmed or refuted [3]. Japan is located between the latitude $26^{\circ}$ to $45^{\circ}$ North [4]. There exist interesting concordance and discordance of clinical features of vasculitis between Japan and Europe/USA. The differences will be reviewed here.

Vasculitis is much more heterogeneous in its clinical features such as incidence, phenotype and/or genotype among areas. As few giant cell arteritis (GCA) patients have been reported in Japan, compared with Europe and the USA [5], the prevalence of GCA in studies in Japan will be also reviewed and discussed. Moreover, differences in renal involvement in WG and MPA between the UK and Japan [6] will also be discussed. These area differences will be reviewed in association with human leukocyte antigen (HLA) such as cANCA-positive WG in 1996 [7], and autoreactive $\mathrm{T}$ cell response MPO fragments $[8,9]$.

A good correlation between the extent of crescent formation and the MPO-ANCA titer has been demonstrated [10]. Differences in reactivity suggest differences of binding to MPO epitopes by MPO-ANCA. The titer of MPOANCA does not always reflect disease activity, and this inconsistency may be attributable to differences in epitopic specificity by MPO-ANCA between patients. We also review the analyses of the epitopes in MPO-ANCA in the Japanese population. Epitopes on MPO recognized by MPO-ANCA from patients with MPO-ANCA-associated vasculitis in the Japanese population have been analyzed using recombinant MPO fragments [11]. Differences in binding specificity may influence the pathogenic potential of the antibodies. The immunodominant epitopes have not been precisely defined. Erdbrügger et al. [12] reported that PR3-ANCA and MPO-ANCA do not interfere with the enzymatic activity of MPO. The differences in binding specificity may influence the pathogenic potential of the antibodies [13]. Van der Geld et al. [14] demonstrated that noncontiguous amino acids are important to the structure of epitopes. In this review, the epitopes in patients with vasculitis in Japan will be discussed. Concomitantly, ELISA systems for three kinds of MPO-ANCA and PR3-ANCA used in Japan have been compared with those commonly used in Europe. The report of Ito-Ihara et al. [15] that described ANCA ELISA systems in Japan will mainly be reviewed.

Finally, we will review Kawasaki disease (KD), a systemic vasculitis in childhood prevailing in Japan that was first described in 1967 by Dr. Tomisaku Kawasaki. The nationwide surveys for KD conducted every 2 years show that the number of KD patients has been increasing [16].
KD is now appearing all over the world, but is most prevalent in Japan and Asian countries [16, 17].

Thus, in this review, we describe ANCA-associated vasculitis (AAV) and other vasculitis including large vessel vasculitis (Takayasu's arteritis, TAK) and GCA and $\mathrm{KD}$ in Japan. Epitopes of MPO-ANCA in vasculitis in the Japanese population and a comparison of ANCA-ELISA systems in Japan and Europe are also described.

\section{Vasculitides in Japan and Europe/USA}

\section{Differences in the Clinical Presentation of Vasculitis} between Japan and Other Countries

It is important to study and understand the concordance and discordance of clinical features of vasculitis between populations in Japan and Europe and the USA. Compared to rheumatoid arthritis (RA) and systemic lupus erythematosus (SLE), vasculitis is much more heterogeneous in its clinical features, such as incidence, phenotype and/or genotype among areas and/or countries.

TAK and GCA are two types of vasculitis where inflammation occurs in the large vessels, and their granulomatous vasculitis with the presence of giant cells [18, 19]. Compared with Europe and the USA, fewer GCA patients and a higher incidence of TAK patients are reported in Japan. The point prevalence of GCA in studies from Japan in 1997 was 690 patients (95\% CI 400-980) [5]. The prevalence of patients aged more than 50 years was 1.48 / million compared with 200 in the USA and 60 in Spain [20, 21].

We have been asking ourselves why there are so few GCA patients in Japan. One factor leading to this question is the fact that we do not frequently see patients with ankylosing spondylitis in Japan, which is not the case in Korea and China. The incidence of HLA-B27 in Japan is only $0.3 \%$ compared with about $5 \%$ in Korea and China and $7-14 \%$ in the USA and Europe [22]. Therefore, genetic factors affecting the incidence of the disease must be unique even among far eastern countries. HLA$\mathrm{DRB1}^{*} 0401$ or HLA-DRB1* 0404 are predominantly (60\%) detected in patients with GCA in the USA; however, HLA-DRB1 ${ }^{*} 0401$ and HLA-DRB1 ${ }^{*} 0404$ are less frequent, 2.9 and $0.7 \%$, respectively, as determined in 493 Japanese healthy individuals compared with 15.9 and $3.2 \%$, respectively, in 60 healthy individuals in the USA [19]. This is one of the reasons why the incidence and/or prevalence of GCA is not high in Japan.

In Japan, TAK is predominant in young female patients and mainly affects the aortic arch (type I), as determined 
Table 1. Point prevalence of vasculitis patients in Japan

\begin{tabular}{|c|c|c|c|c|}
\hline $\begin{array}{l}\text { Year of } \\
\text { investigation }\end{array}$ & Disease & $\begin{array}{l}\text { Estimated number } \\
\text { of patients }\end{array}$ & $\begin{array}{l}\text { Average age of patients at } \\
\text { the time of study, years }\end{array}$ & $\begin{array}{l}\text { Male/ } \\
\text { female }\end{array}$ \\
\hline 1993 & Takayasu's arteritis & 4,800 & $35-65$ & $1 / 10$ \\
\hline \multirow[t]{5}{*}{1994} & Polyarteritis nodosa & 1,400 & 56.2 & $1 / 1.1$ \\
\hline & Wegener's granulomatosis & 670 & 46.2 & $1 / 1.2$ \\
\hline & Allergic granulomatous angiitis (Churg-Strauss syndrome) & 450 & 47.1 & $1 / 1.1$ \\
\hline & Buerger's disease & 10,000 & $45-65$ & $9.7 / 1$ \\
\hline & Malignant rheumatoid arthritis & 4,200 & 53 & $1 / 2.2$ \\
\hline \multirow[t]{2}{*}{1998} & Giant cell arteritis (temporal arteritis) & 690 & 62.9 & $1 / 1.6$ \\
\hline & ANCA-associated vasculitis & 2,700 & 59 & $1 / 1.8$ \\
\hline
\end{tabular}

by angiography. The patients show significantly high levels of HLA-B52 (56\%) and HLA-B39 (17\%) compared with healthy controls (25 and 6\%, respectively) [23]. However, it was reported that a larger number of patients with TAK in India and other East Asian countries are middle-aged males with affected abdominal aortas (type III), and who have HLA-B39 [23]. A nationwide point prevalence survey in the form of a retrospective, hospital-based study was conducted in 1994 and 1998 (table 1). Although the data are not new and do not show the real incidence, the prevalence of vasculitis/vasculopathy in Japan understandable. Buerger's disease and TAK are common, but WG and GCA are not frequently observed in Japan.

In 1997, we conducted a retrospective, hospital-based, nationwide survey on Japanese patients with AVV. The survey reported $63 \mathrm{MPA}, 28 \mathrm{WG}$ and 12 Churg-Strauss syndrome (CSS) patients and 104 patients with undifferentiated AAV (most patients were renal-limited vasculitis of MPA analyzed by the records). The point prevalence of AVV in the 1997 survey demonstrated that MPA is the most common AVV in Japan. Among 207 AVV patients, PR3-cANCA and MPO-pANCA were demonstrated in 26.3 and $80.8 \%$, respectively. Among 28 WG patients, PR3-cANCA and MPO-pANCA were shown in 86 and $14 \%$, respectively; and among 63 patients with MPA in 22 and $87 \%$ of patients, respectively. For MPA, prominent manifestations were renal involvement (87.3\%), mainly rapidly progressive glomerulonephritis (RPGN), and pulmonary involvement (63.5\%) including interstitial pneumonia/pulmonary fibrosis (33.3\%) and pulmonary hemorrhage (22.2\%). Patients with MPA having only a pulmonary localized lesion have been reported. In addition, 93\% of Japanese WG patients demonstrated the ear, nose and throat (ENT) features, whereas only 39\% demonstrated renal involvement. Compared to WG patients in Europe and the USA, renal involvements are not common in Japanese WG patients. Furthermore, positive rate and titer of PR3-/cANCA are relatively low in WG patients who have localized ENT lesions. These results were included in the annual report for the Research Committee of Intractable Vasculitis, the Ministry of Health and Welfare, Japan, in 1998 by Hashimoto and colleagues.

The question why MPA is more common than WG in Japan while WG is the most common AVV in Europe and the US is important. This question led us to start an epidemiological study on Japanese patients with AVV in collaboration with the European Systemic Vasculitis Study Group (EUVAS) members Drs. D. Jayne, R. Watts, D. Scott, N. Rasmussen and U. Specks. The understanding of differences will lead to new insights into the etiology and pathogenesis of vasculitides.

\section{Latitude of Japan}

Japan is located between the latitude $26-45^{\circ}$ North. Asahikawa city $\left(43.5^{\circ}\right.$ North) on Hokkaido island is close to the latitude of Lugo, Spain $\left(42^{\circ} \mathrm{N}\right)$. This is compatible with the latitude theory of AAV [4] (fig. 1).

Since we had to update old epidemiological data for AAV and determine the present incidence of AAV in Japan, we are glad to have started the new epidemiological study with UK/EUVAS members. Results of incidence of AAV in Miyazaki Prefecture are described in the section 'Primary Renal Vasculitis in Japan'.

It is interesting to note that a study from Beijing, China, demonstrated that $60.7 \%(54 / 89)$ of patients with WG were MPO-ANCA positive, 38.2\% (34/89) were PR3ANCA positive, and patients with MPO-ANCA had multisystem involvement and elevated initial serum creatinine level as compared with PR3-ANCA-positive WG patients $[24,25]$. 
Fig. 1. Geographic difference in latitude between Japan and Europe.

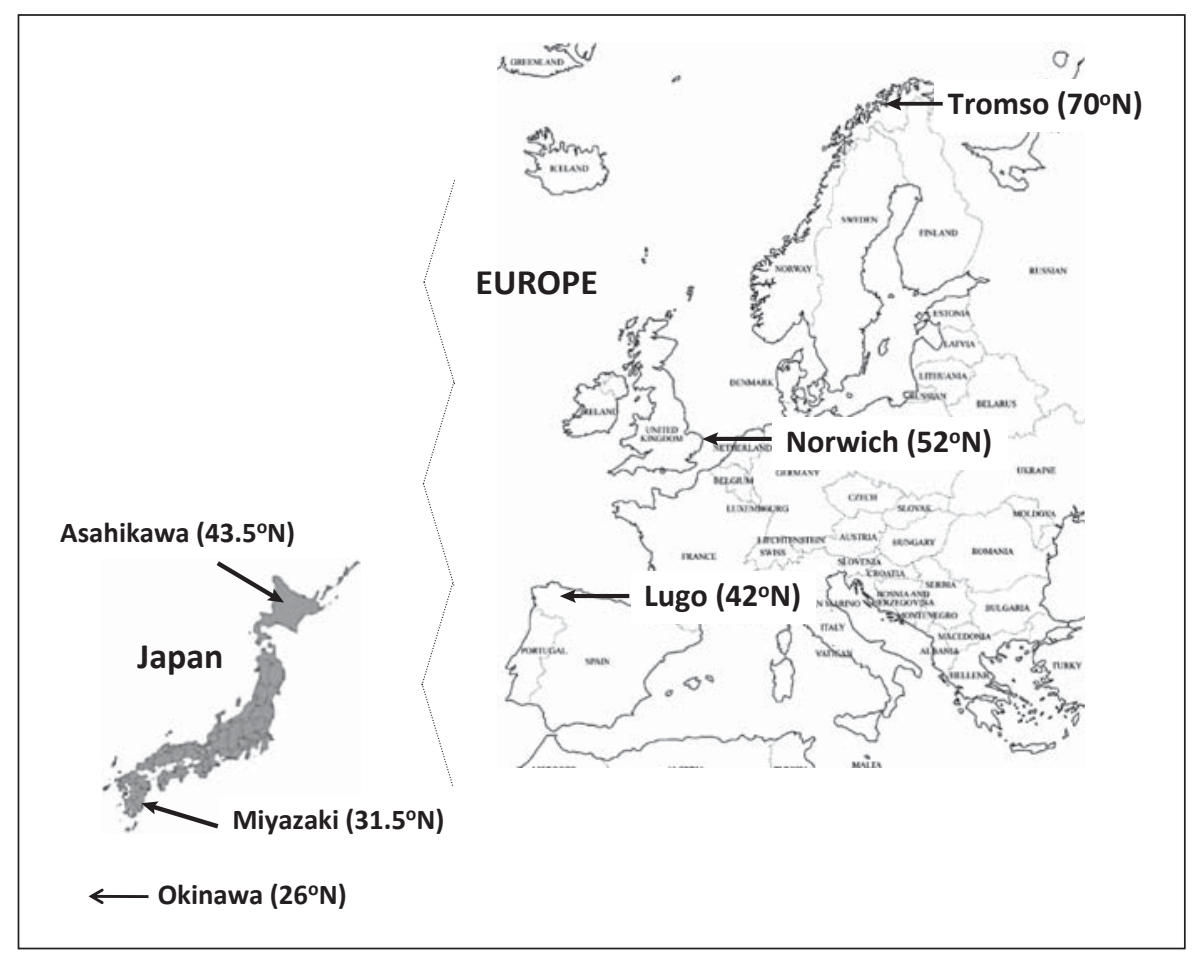

\section{Phenotype}

The difference in the clinical presentation of TAK arteritis between Japan and countries in East Asia has been mentioned above; therefore, we will now discuss the differences in clinical presentation of patients with GCA between Japan and other countries. Our study demonstrates that no remarkable differences were found in the clinical features of patients with GCA between Japan and other countries, although GCA patients are not commonly found in Japan compared with the USA and Europe [19]. Further, differences in renal involvement in WG and MPA between the UK and Japan were demonstrated by Watts et al. [6]. The data that patients having a localized type of WG are more frequently found in Japan than WG patients with renal involvement were supported by Dr. Harabuchi at Asahikawa Medical University. A report from other ENT doctors with the same conclusion was published recently [26]. In fact, renal involvement was demonstrated in $12-40 \%$ of 21 WG patients in two reports. In another hospital-based, nationwide, retrospective study in Japan in 1988 and 1998, 39-63\% of 172 patients had renal involvement. Renal involvement was found in $77 \%$ of $158 \mathrm{WG}$ patients and $77 \%$ of $70 \mathrm{WG}$ patients by Drs. Gross and Hoffman, respectively [27].

\section{Genotype}

A significant association of HLA-DRB1*0901 with MPA ( $p=0.037$, OR 2.44, 95\% CI 1.33-4.46) as well as with MPO-ANCA positivity $(\mathrm{p}=0.014$, OR $2.44,95 \%$ CI $1.41-$ 4.22) was demonstrated by Tsuchiya and colleagues [2831]. In their paper from 2003 [28], the Japanese diagnostic criteria for MPA proposed by the Research Committee on Intractable Vasculitides in 1998 were described. An interesting report regarding the association of HLA-DR9 in cANCA-positive WG $(62.5 \%, 10 / 16$ patients as compared with $26 \%$ of healthy controls; $\mathrm{p}<0.05)$ was reported in 1996 [7]. The decreased activation potential of natural killer cells and/or T cells associated with killer cell immunoglobulin-like receptor/HLA genotypes was demonstrated in MPA patients, and insufficient resistance against infections in MPA patients was suggested [31]. Autoreactive T cell response to the MPO fragments was found, and several distinct epitopes on MPO fragments recognized by HLA-DR-restricted CD4+ T cells were demonstrated $[8,9]$.

\section{Primary Renal Vasculitis in Japan}

\section{Experience of Vasculitis in Japan}

We sometimes come across cases with MPA involving the kidney, most of which are MPO-ANCA positive. 
Table 2. Difference in ANCA phenotype in patients with crescentic glomerulonephritis/microscopic polyangiitis/renal limited vasculitis between Japan and other countries

\begin{tabular}{lrll}
\hline Country & Patients & $\begin{array}{l}\text { Positive } \\
\text { MPO-ANCA, \% }\end{array}$ & $\begin{array}{l}\text { Positive } \\
\text { PR3-ANCA, \% }\end{array}$ \\
\hline USA [34] & 107 & 64 & 36 \\
EU [35] & 80 & 63 & 25 \\
Sweden [36] & 99 & 48 & 32 \\
UK [37] & 153 & 65 & 25 \\
Japan [38] & 63 & 79 & 13 \\
Japan [39] & 369 & 90 & 8 \\
Japan [40] & 993 & 89 & 6 \\
\hline
\end{tabular}

On the other hand, we rarely have the cases with PR3ANCA-positive, crescentic glomerulonephritis and/or WG with renal involvement. However, in the European and American articles, much higher rates of the patients with positive PR3-ANCA and/or WG are shown among those with RPGN or AAV compared to Japan. In this section, we describe the epidemiology, the characteristics and outcome of ANCA-associated primary renal vasculitis (PRV): MPA, WG, and CSS with renal involvement, and renal limited vasculitis (RLV) in Japan comparing to those of European countries and the USA. Patients with PRV are defined according to the following criteria in accordance with EUVAS [32]: new patients with MPA, WG, CSS or RLV and renal involvement (elevated serum creatinine, hematuria, proteinuria, or red cell casts) attributed to active vasculitis with or without other organ involvement.

\section{Epidemiology of PRV in Japan}

In a nationwide, retrospective, hospital-based survey on RPGN recently conducted by the Japanese Society of Nephrology [33], the most frequent primary disease was RLV (42.1\%), the second was MPA (19.4\%) and the third was anti-glomerular basement membrane (anti-GBM) associated RPGN (6.1\%). MPO-ANCA was positive in $88.1 \%$ of RLV patients and $91.8 \%$ of MPA patients. On the other hand, the prevalence of WG was only $2.6 \%$ among the total RPGN patients $(\mathrm{n}=1,772)$, and the positive rate of PR3-ANCA among patients with RLV was 7.4\%, that of MPA was $6.1 \%$, and that of WG was $71.1 \%$. These data were not different from previous studies in Japan. European and American studies revealed a third of RPGN/ MPA/RLV patients were PR3-ANCA positive, which is clearly higher compared to Japan (table 2).
Until recently, the incidence, but not prevalence, of AAV has not been determined in Japan. We conducted the first population-based survey of PRV in Miyazaki Prefecture between 2000 and 2004 [2] based on recent epidemiological methods [41] and the sub-classification of AAV by the EUVAS [32]. Among 56 identified patients, 91\% were MPO-ANCA positive and none had WG or CSS. The male/female ratio was $24 / 32$, and the average age was $70.4 \pm 10.9$ (mean \pm SD) years. The estimated annual incidence of PRV was 14.8 (95\% CI 10.8-18.9) and 44.8 (95\% CI 33.2-56.3) per million adults (over 15 years old) and seniors (over 65 years old), respectively. This value was reported to be 12.4 in Bristol, UK [42], 18 (95\% CI 13-24) in Norfolk, UK [41], and 16 (95\% CI 12-31) in Orebro, Sweden [36]. Thus, the annual incidence of PRV seems to be similar in Japan and Europe.

The race and genetic background of the patients should be closely related to the differences in rates of MPOANCA versus PR3-ANCA between Japan and Europe [28]. A geographic difference in the incidence of systemic vasculitides has also been suggested. The incidence of WG and MPA might be latitudinal; WG is frequently seen in high-latitude areas such as Sweden and the UK, while MPA in low-latitude areas such as Bahrain and Miyazaki. On the other hand, examination of the type of ANCA in sera from patients with AAV revealed that the results of commercially available ELISA kits used in EUVAS and Japan did not differ [15].

\section{Clinical Phenotype and Features}

Watts et al. [6], collaborators of Japan-UK vasculitis epidemiology study, have recently compared the incidence of PRV in the UK with Japan during the period 2000-2004 using the same case definitions. As shown in table 3 , the underlying disease was very different, with MPA/RLV predominating in Japan, although the overall occurrence of PRV was similar in Japan and the UK. ENT and neurological involvement were much less common in Japan. ANCA status was also different between Japan and the UK; positive MPO-ANCA was 91.1 versus $55.6 \%$, and positive PR3-ANCA 0 versus 33.3\%. Thus, PRV patients with cANCA/PR3 and/or WG seem to be much less common in Japan than the UK.

\section{Outcome and Treatment}

To improve the survival rate and renal prognosis in patients with AAV/PRV, the therapeutic methods may be different between Japan and Europe/USA because clinical phenotype and features were very different among 
Table 3. Comparison of epidemiology and clinical features of ANCA-associated renal vasculitis between Japan and the UK

\begin{tabular}{lccc}
\hline & Japan (Miyazaki) & UK (Norfolk) & $\mathrm{p}$ \\
\hline Male/female & $24 / 32$ & $13 / 14$ & \\
Mean age, years & 70.4 & 63.5 & \\
Incidence, /million & & & \\
$\quad$ Total & $14.8(10.8-18.9)$ & $12.2(8.0-17.7)$ & \\
$\quad$ MPA/RLV & $14.8(10.8-18.9)$ & $5.0(2.4-8.8)$ & \\
WG & 0 & $5.8(2.9-9.4)$ & $<0.001$ \\
CSS & 0 & $1.4(0.3-3.9)$ & $\mathrm{NS}$ \\
ENT & $1(1.8 \%)$ & $18(66.6 \%)$ & $<0.02$ \\
Respiratory & $23(41.1 \%)$ & $11(40.7 \%)$ & $\mathrm{NS}$ \\
Nervous & $3(5.4 \%)$ & $8(29.8 \%)$ & $<0.001$ \\
Gastrointestinal & $2(3.6 \%)$ & $3(11.0 \%)$ & $<0.001$ \\
MPO-ANCA & $51(91.1 \%)$ & $9(35.5 \%)$ & $\mathrm{NS}$ \\
PR3-ANCA & 0 & $2(7.4 \%)$ & \\
Negative ANCA & $5(8.9 \%)$ & & \\
\hline
\end{tabular}

these countries. Although the combined therapy with cyclophosphamide and glucocorticoids is recommended as the standard of care in remission induction therapy in Europe and the USA, only one fourth to one third of PRV/RPGN patients have received these drugs in Japan $[33,43]$. Both patient and renal survival rates among Japanese PRV patients (RLV + MPA + WG) recently improve to 84.1 and $83.7 \%$ at 12 months, and to 79.1 and $78.5 \%$ at 24 months, respectively [33]. A major cause of death is indirectly related to vasculitis and mostly consists of treatment-related infectious complications. This could be because a high proportion of Japanese patients are elderly and/or dialysis-dependent at presentation with MPA/RLV predominating in Japan [43]. In the Japanese clinical guideline for RPGN based on the questionnaire survey [33], milder immunosuppressive treatment such as an initial oral prednisolone dose reduction $(0.6 \sim 0.8 \mathrm{mg} / \mathrm{kg} / \mathrm{day})$ with or without immunosuppressant is recommended for older and/or dialysis-dependent patients.

On the other hand, randomized clinical trials of new therapies for AAV have been performed in Europe and the USA. On the principle that more severe disease requires more aggressive therapy, EUVAS devised a system for subgrouping AAV presentations, for example, based on the severity of renal impairment, for different regimens [44]. Results of such trials are very informative and might lead to consensus recommendations on how to AAV/PRV should be treated depending on the types, the disease severity and age.

AAV, Large Vessel Vasculitis and Kawasaki Disease in Japan

\section{Laboratory Investigations: ANCA Epitopes in the Japanese Population and ELISA Evaluation}

\author{
Epitopes of MPO-ANCA in Vasculitis in the Japanese \\ Population \\ MPO-ANCA-Associated Vasculitides in Japan \\ ANCA are found in a high percentage of patients with
} WG, MPA, and CSS and are used as diagnostic markers for these diseases, which are also termed AAV. Furthermore, MPO-ANCA has been demonstrated to be a good marker for the diagnosis of these disorders. The titer of MPO-ANCA is correlated with the activity of autoimmune, necrotizing, crescentic glomerulonephritis. In addition, a good correlation between the extent of crescent formation and the MPO-ANCA titer has been demonstrated [10]. However, a low titer of MPO-ANCA is found in the sera from some patients with active crescent formation, and conversely, high titer can be found during the remission stage in Japanese patients. Therefore, it seems that the titer of MPO-ANCA is not always correlated with the activity of the disease due to the difference in reactivity of MPO-ANCA to the MPO molecule. Such differences in reactivity suggest differences of binding to MPO epitopes by MPO-ANCA. The inconsistency of MPOANCA titer in primary systemic vasculitis (PSV) may be attributable to differences in epitopic specificity by MPOANCA between patients. 
Fig. 2. Frequency of epitope sites of MPOANCA in the serum of patients with vasculitis. Incidence in each vasculitis shows relative ratio (\%) in all epitopes reacted with MPO fragments [45].

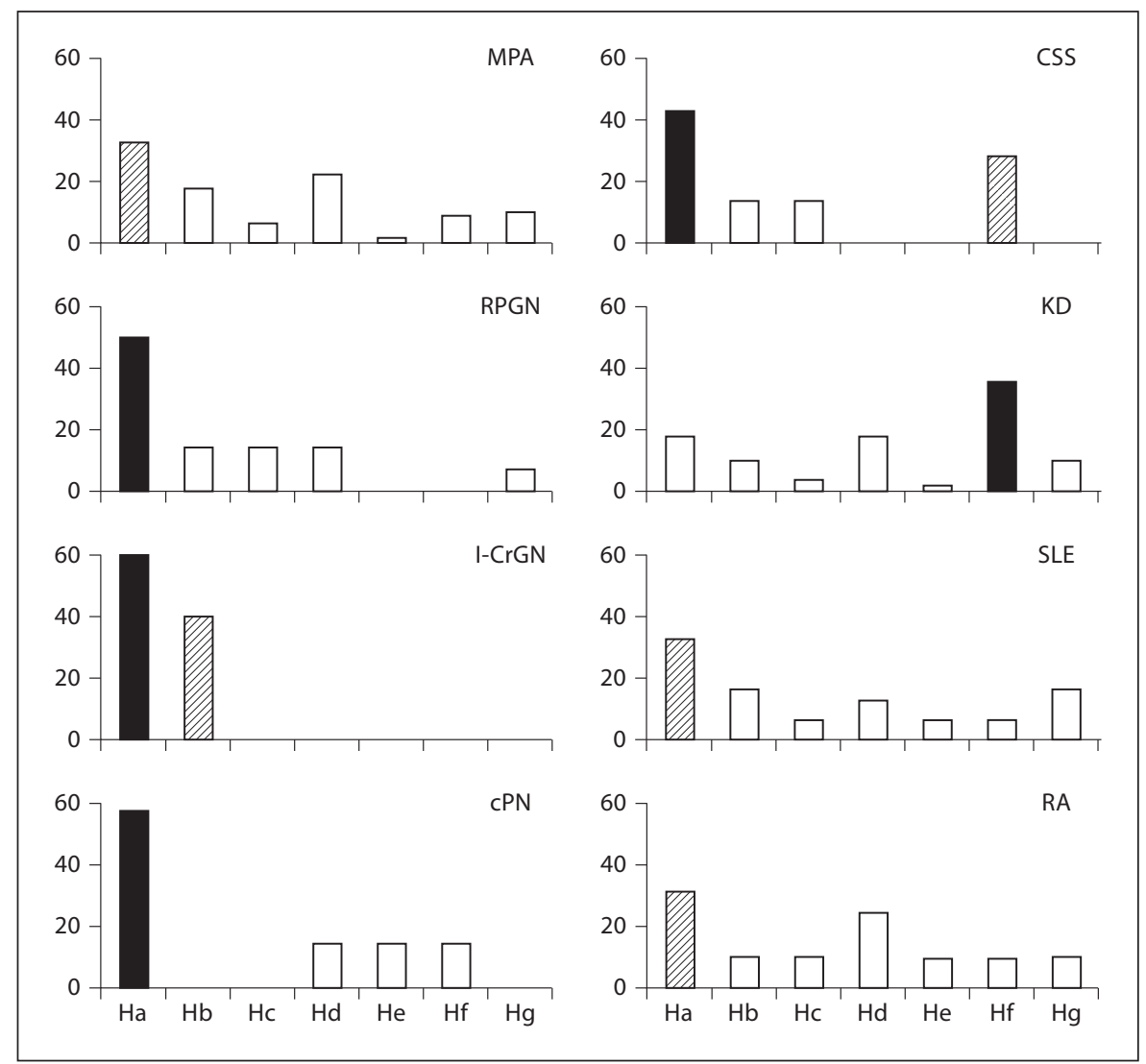

Analyses of the Epitopes in MPO-ANCA in the Japanese Population

Epitopes on MPO recognized by MPO-ANCA from patients with MPO-AAV in the Japanese population have been analyzed using recombinant MPO fragments set using deletion mutants of MPO composed of eight fragments of the heavy-chain subunit, and two fragments of the light chain subunit expressed in Escherichia coli [11].

Epitope analysis may also explain the occurrence of MPO-ANCA in different vasculitic syndromes. The sera of $148 \mathrm{MPO}$-ANCA-positive patients from four vasculitic syndromes (MPA, classic polyangiitis nodosa, CSS and KD) and from patients with RA and SLE are applied to the epitope analysis. The sera have been collected by the Intractable Vasculitis Research Project Group in Japan.

In PSV, MPO-ANCA are markers for diagnosis and have been implicated in pathogenesis of vasculitis. Although high MPO-ANCA titers are associated with an increased risk of disease activity, MPO-ANCA titers do not necessarily correlate with disease activity or vasculitic syndrome. The severity of the diseases in MPA with renal involvement is correlated with particular epitopes of MPO-ANCA recognizing the $\mathrm{N}$ or $\mathrm{C}$ terminus of the MPO heavy chain [9]. The epitopes recognized by MPOANCA from sera of patients with four vasculitic syndromes (MPA, cPN, CSS, KD) and from patients with RA and SLE in hospitals at Juntendo University, Kyorin University, Kyoto University, Tokyo Medical School, Fujita Medical Health University, and Hiroshima City in Japan, members of the project funded by the Ministry of Health, Labour and Welfare, have been analyzed [45].

No serum showed epitopes La and Lb of light chain MPO, and sera of $68.6 \%$ of patients showed a positive reaction to one or more epitopes of heavy-chain MPO. An analysis of the binding level showed that MPA sera mainly reacted to the $\mathrm{Ha}$ epitope at the $\mathrm{N}$ terminus of the MPO heavy chain, CSS sera reacted to the Ha and $\mathrm{Hf}$ epitopes close to the $\mathrm{C}$ terminus of the MPO heavy chain, and KD reacted mainly to Hf, whereas SLE and RA sera reacted to all epitopes. These results suggest that MPO-ANCA recognizing specific regions of the N terminus of the MPO $\mathrm{H}$ chain confer an increased risk of vasculitis such as MPA and CSS. Furthermore, the epitopic specificity of MPO-ANCA differentiates vascu- 
litic from nonvasculitic syndromes associated with MPO-ANCA positivity and differentiates between vasculitic syndromes (fig. 2) [45].

MPO-ANCA is a major AAV pathogen $[46,47]$ and recognizes different binding sites (epitopes) on its corresponding antigens. Differences in binding specificity may influence the pathogenic potential of the antibodies. We previously reported relevant data between the MPOANCA risk epitope(s) and high-titer sera of MPOANCA patients $[9,11]$, but controversy remains as to MPO epitope sites $[14,45]$. The immunodominant epitopes have not been precisely defined. Erdbrügger et al. [12] reported that PR3-ANCA and MPO-ANCA do not interfere with the enzymatic activity of MPO, suggesting that a major epitope may be close to the last half of the heavy chain, the reactive sites in our results. However, the correlation between epitopes and vasculitis is not understood. The differences in binding specificity may influence the pathogenic potential of the antibodies [13]. Furthermore, we have reported a human MPO epitope using 3-D human MPO surface data (Protein Data Bank 1CXP) [48]. Van der Geld et al. [14] showed that noncontiguous amino acids are important to the structure of epitopes.

\section{Comparison of ANCA-ELISA Systems in Japan and Europe}

In Japan, three kinds of MPO-ANCA and PR3-ANCA ELISA systems are currently available as extracorporeal diagnostic agents authorized by the Ministry of Health and Welfare of Japan. Comparison of these ELISA systems with those commonly used in Europe is essential for international collaboration and for epidemiological and clinical research. However, the major problem with the current application of ANCA ELISA systems is the lack of both international standard sera and international standardization of assay systems. Commercially available ELISA systems have a wide range of performance characteristics and employ arbitrary units determined by each manufacturer [49]. When interpreting an ANCA test, therefore, the clinician must take into account the differences between the ELISA systems. Ito-Ihara et al. [15] have compared the sensitivity and specificity of two major commercially available ANCA ELISA systems in Japan and one of the most widely used systems in Europe for MPO-ANCA and PR3-ANCA using plasma obtained from Japanese patients with a clinical and histological diagnosis of WG, MPA, or CSS. In addition, the correlation of ANCA values among ELISA systems has been also assessed.

AAV, Large Vessel Vasculitis and Kawasaki Disease in Japan
Table 4. Correlation coefficient between MPO-ANCA ELISAs

\begin{tabular}{llll}
\hline MPO-ANCA ELISAs & Nipro & MBL(BS) & Wieslab \\
\hline Nipro & - & 0.891 & 0.879 \\
MBL & 0.891 & - & 0.899 \\
Wieslab & 0.879 & 0.899 & - \\
\hline
\end{tabular}

Samples with PSV before treatment and in the follow-up period $(n=146)[15]$.

\section{Japanese Patient Population and Diagnostic Criteria} for Comparison of ELISA Kits

The plasma samples derived from newly diagnosed patients with PSV including WG, MPA, or CSS in accordance with the American College of Rheumatology classification criteria and Chapel Hill consensus criteria with reference of EMEA algorithm method are accessed. A modified algorithm to be irrespective of positivity of ANCA has been applied. All patients were systematically assessed for potential subclinical granulomatous disease with diagnostic imaging as well as having ENT consultation. Confirmatory organ histological biopsies have been available in 8 of 11 patients with WG, including four renal biopsies, two lung biopsies, and two biopsies from nodules of paranasal sinuses. Renal biopsies have been performed in all the MPA and CSS patients and revealed that all of them showed renal involvement.

\section{Comparison of MPO-ANCA ELISAs}

Correlations between MPO-ANCA ELISAs are analyzed using 146 plasma samples from patients with PSV at various stages (MPA, $n=132$; CSS, $n=1$; WG, $n=13$ ) [15]. All data show $\log _{10}$ transformed to normalize distributions prior to these analyses. Nipro MPO-ANCA ELISA has been positively correlated with MBL MPO-ANCA $(\mathrm{r}=0.891, \mathrm{p}<0.0001)$ and Wieslab MPO-ANCA $(\mathrm{r}=0.879$, $\mathrm{p}<0.0001)$. MBL MPO-ANCA and Wieslab MPO-ANCA are positively correlated with each other $(\mathrm{r}=0.899, \mathrm{p}<$ 0.0001; table 4). For example, Nipro MPO-ANCA and MBL MPO-ANCA are widely used in reference laboratories or university hospitals in Japan. Sensitivity and specificity are not different between these two systems, and they have been positively correlated with each other (fig. 3). Holle et al. [49] have analyzed ANCA ELISAs from eleven manufacturers and reported that applying the manufacturers' cutoff values results in great variation in sensitivity. Lowering the cutoff values increased the sensitivity and

Kidney Blood Press Res 2010;33:442-455 
reduced specificity but increased overall diagnostic performance. They have concluded that the low sensitivity of some commercial ELISA systems reflects the high cutoff values rather than methodological problems in the assays.

Thus, the two major MPO-ANCA ELISA systems commercially available in Japan exhibit high sensitivity and specificity and have a diagnostic value comparable to that of the ELISA systems used in Europe. Based on the comparison of ANCA testing in Japan, this will facilitate future international surveys exploring differences in the epidemiology of PSV and etiological factors contributing to its pathogenesis.

\section{Comparison of PR3-ANCA ELISAs}

Nipro PR3-ANCA is equivalent to Immunoscan PR3ANCA (Euro-Diagnostica) and MBL PR3-ANCA is equivalent to BINDAZYME ${ }^{\mathrm{TM}}$ Human Anti-PR3 Enzyme Immunoassay kit (Binding Site). These kits are used internationally and have already been well screened throughout the world. Trevisin et al. [50] reported that the sensitivity of Euro-Diagnostica Immunoscan PR3ANCA is $90 \%$, with $96 \%$ specificity. Binding Site BINDAZYME RP3-ANCA ELISA has reported 60-96\% sensitivity and $88-100 \%$ specificity [50-52]. Then, it would be possible to more directly compare the performance of ELISAs for WG between Europe and Japan. In the meantime, before the results become clear, it is important that laboratories understand the differences and limitations of the assays. One limitation of our study is the small number of WG samples in Japan.

\section{Analysis and mRNA Diagnostic System for Vasculitis}

Microarray analysis of mRNA from PBMC has been strongly promoted by Kobayashi and colleagues [53, 54] in their large-scale gene profiling study to find sensitive and specific DNA markers for diagnosing vasculitis.

\section{Model Mice for ANCA-Associated Vasculitis}

To clarify the mechanisms of crescentic formation in $\mathrm{MPO}-\mathrm{AAV}$, spontaneous crescentic glomerulonephritisforming mouse/Kinjoh (SCG/Kj) mice have been designed in Japan [55]. SCG/Kj is a recombinant congenic strain of mice that was genetically segregated from BXSB $\times$ MRL/Mp-Faslpr (MRL/lpr)F1 mice by selected brother $\times$ sister matings [51]. SCG/Kj mice also develop antiMPO antibody $[56,57]$ and MPO acts as a driving antigen in the anti-MPO response observed in SCG/Kj mice [58]. Hamano et al. [59] investigated related traits susceptible to aberrant production of MPO-ANCA by genome-wide quantitative trait locus mapping in SCG/Kj mice.

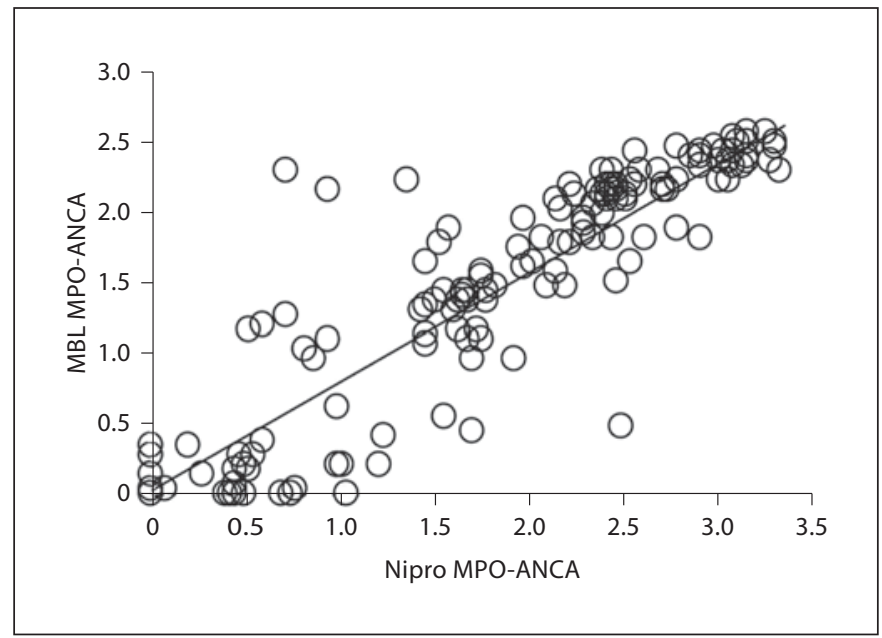

Fig. 3. Correlation between Nipro MPO-ANCA and MBL MPOANCA. Correlations between MPO-ANCA were analyzed using 146 plasma samples from patients with PSV in the active and follow-up stages (MPA, $n=132$; CSS, $n=1$; WG, $n=13$ ) [15].

\section{Kawasaki Disease}

KD was first described in 1967 by Dr. Tomisaku Kawasaki as mucocutaneous lymph node syndrome. The etiology of this disease remains unknown, and the disease affects most commonly infants and young children $[60,61] . K D$ is considered a kind of systemic vasculitis syndrome, and it invades primarily medium-sized muscular arteries. This disease has attracted special interest, because death from this disease is most frequently attributable to ischemic heart disease in children caused by thrombosed coronary artery aneurysms, secondary to coronary arteritis.

\section{Epidemiology}

In Japan, nationwide surveys have been conducted every 2 years since 1970 . The age distribution at onset of KD shows a peak at 9-11 months, and $70 \%$ of all KD occurs in patients younger than 3 years. The male/female ratio among patients with $\mathrm{KD}$ is $1 / 3$. According to the latest survey, the total number of registered patients is over 240,000 . The number of newly diagnosed patients is more than 10,000 per year. The number of KD patients has been increasing, and the incidence of KD in children is increasing further due to the low birthrate [16] (fig. 4). Acute-stage cardiac abnormalities show an incidence of about $13 \%$, and the incidence of cardiac sequelae is about $4 \%$. The mortality rate has markedly fallen to $0.01 \%$. 
Fig. 4. Incidence of patients with KD in Japan by year [16].

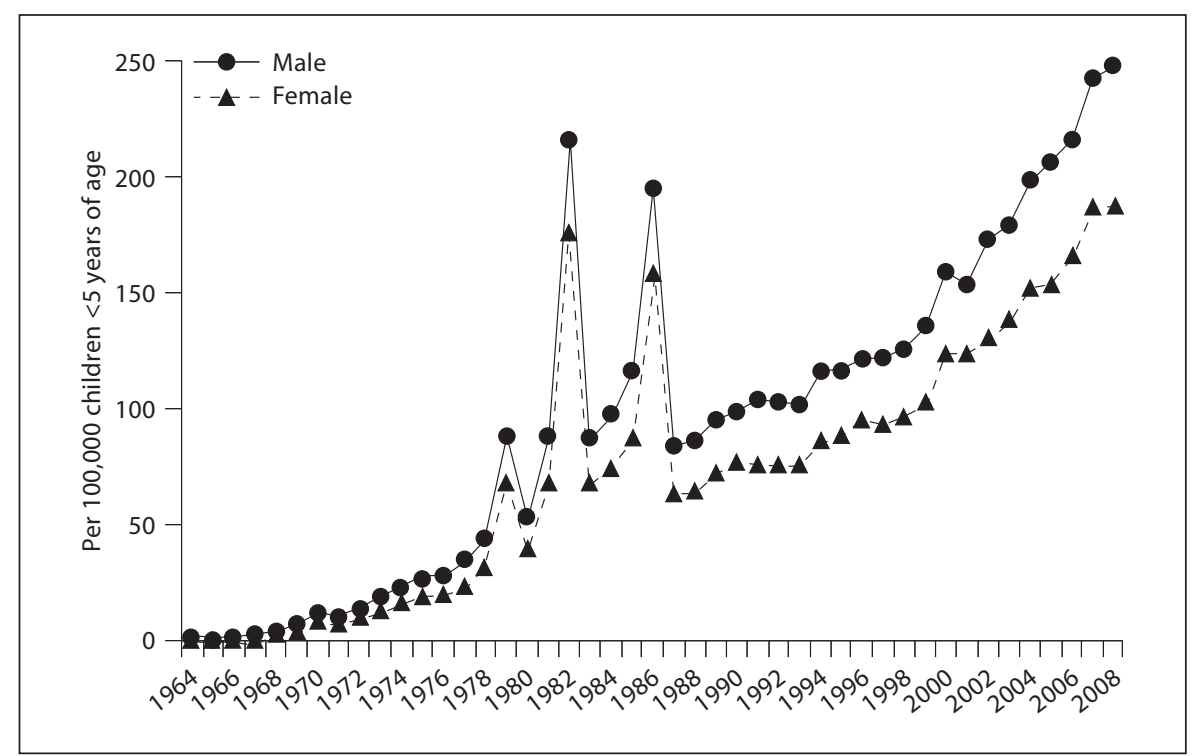

$\mathrm{KD}$ is now appearing all over the world, but is most prevalent in Japan and Asian countries. The incidence in children $<5$ years old is $216.8 / 100,000$ in Japan, 104.6 in Korea, 66 in Taiwan, 53.0 in Hong Kong, 55.1 in China, 24.3 in Canada, 19.6 in the USA, 8.3 in England, 10.0 in Finland. In addition, according to reports from $\mathrm{Ha}-$ waii, USA, the rate in Hawaii is 38.7 , but obviously differs by race; 366.8 for Japanese, 94.8 for Chinese, 76.5 for Hawaiians, 55.7 for Filipino and 7.3 for Caucasians [16, 17].

\section{Diagnosis}

Diagnosis of KD is based on clinical signs and symptoms, which are classified as principal clinical findings, and other clinical and laboratory findings [62]. The principal symptoms include: (1) fever persisting for 5 days or more; (2) bilateral conjunctival congestion; (3) changes in the lips and oral cavity: reddening of the lips, strawberry tongue, diffuse injection of the oral and pharyngeal mucosa; (4) polymorphous exanthema; (5) changes in the peripheral extremities: (initial stage: reddening of the palms and soles, indurative edema; convalescent stage: membranous desquamation from the fingertips, and (6) acute nonpurulent cervical lymphadenopathy. At least five of the above six items must be satisfied for a diagnosis of KD. However, patients having four of the principal symptoms can be diagnosed as having $\mathrm{KD}$ when a coronary aneurysm or dilation is detected by 2-D echocardiography or coronary angiography.

AAV, Large Vessel Vasculitis and Kawasaki Disease in Japan

\section{Pathology}

Coronary arteritis in KD begins as edematous dissociation of the tunica media 6-8 days after the onset of KD. On about the 10th day of the disease, lymphocyte and macrophage infiltration into the arterial wall from the luminal and adventitial sides begins, leading immediately to inflammation of all layers of the artery. The inflammation spreads completely around the artery, and the internal elastic lamina, smooth muscle cells of the media, and other structural components of the artery undergo intense damage; the artery then begins to dilate. Aneurysms develop on about the 12th day after onset when the damage is severe. The blood eddies in the aneurysm making it easy for thrombi to form, and thrombotic occlusion is found in the coronary artery aneurysm of many autopsies of acute-stage KD patients (fig. 5). Arteritis in KD is characterized by proliferative granulomatous inflammation that consists of marked accumulation of monocytes/ macrophages, and aberrant activation of those macrophages is thought to be involved in the formation of vascular lesions $[63,64]$. However, the lesions in the initial stage of inflammation contain not only macrophages and lymphocytes but also many neutrophils [65]. Inflammatory cell infiltration continues until about the 25 th day of the disease, after which the inflammatory cells gradually decrease in number and are almost completely gone by about the 40th day of the disease. Scar from inflammation remain for a long time thereafter. It has been shown that, in the case that a giant aneurysm remains or a vessel recanalization occurs after a thrombotic occlusion of an- 


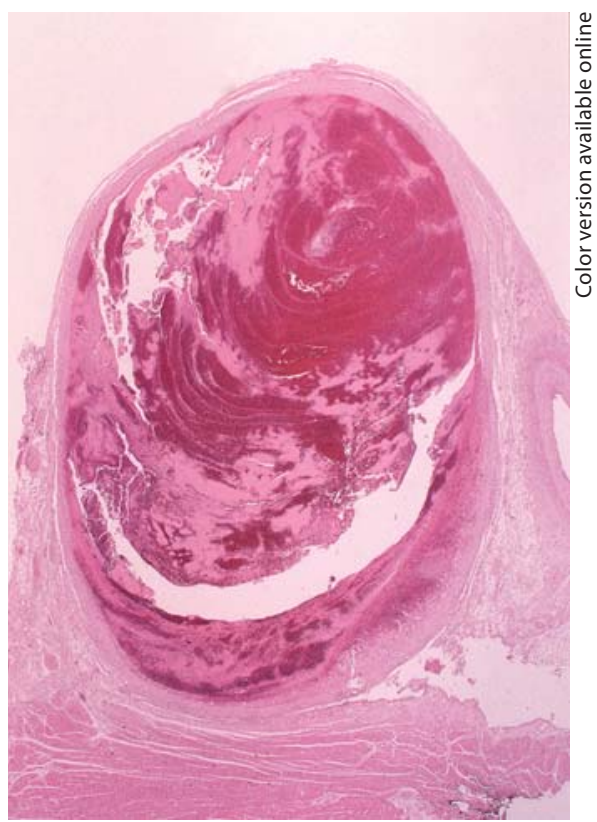

Fig. 5. Low-power view of coronary artery aneurysm. The lumen of the artery is occluded by fresh thrombi.

eurysm, remodeling of the vascular structure, such as reocclusion, continues even in the remote stage [66] (fig. 6). Thus, for patients in whom a giant aneurysm forms during the acute stage, strict countermeasures will be required in relation to ischemic heart disease.

\section{Treatment}

The efficacy of high-dose intravenous immunoglobulin (IVIG) treatment for acute-stage KD patients has become widely recognized $[67,68]$, and today immunoglobulin is administered to $85 \%$ of children with acute-stage KD. IVIG has drastically reduced not only the incidence of coronary artery disorders but also the mortality rate due to KD [16]. However, in about $20 \%$ of patients, even IVIG does not show a good therapeutic effect, with $15 \%$ of patients developing transient dilation or other acutestage coronary artery complications. Although steroids $[69,70]$, neutrophil elastase inhibitors [71], plasma-exchange therapy [72] and anti-cytokine antibody therapy [73] have been tried, the additional or alternative management of KD patients who are nonresponsive to IVIG remains controversial.

\section{Animal Models of KD Vasculitis}

Several animal models of vasculitis in which coronary arteries are often involved have been reported [74-76].

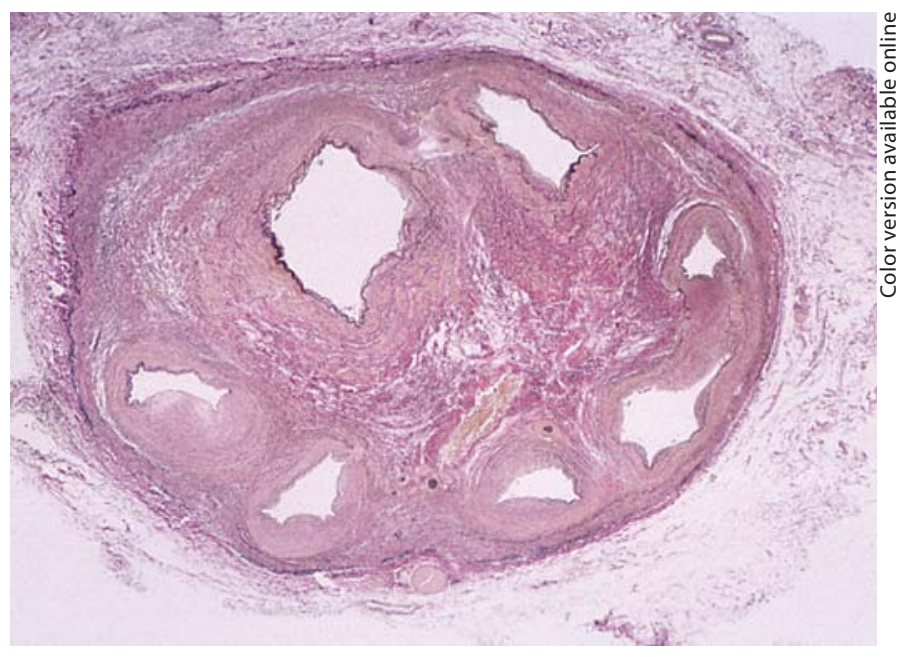

Fig. 6. Recanalized vessels after thrombotic occlusion of coronary artery aneurysm of a KD patient who died 12 years after onset.

Candida albicans cell extract (CADS) from the stool sample of a KD patient was reported to induce murine vasculitis similar to KD coronary arteritis [74]. In an additional study on Candida-derived agents, C. albicans watersoluble fraction (CAWS) induced severe vasculitis at a higher rate of incidence than CADS in many mouse strains, and there are reports on this valuable animal model system in research on systemic immune responses and inflammatory vascular disease [77-79]. The CAWSinduced coronary arteritis is a good model for the histological observation of KD.

\section{Acknowledgements}

The authors thank Drs. H. Hashimoto, E. Muso, H. Nunoi, T. Nagao, K. Tomizawa, T. Ito-Ihara, Y. Hamano, K. Yamazaki, M. Gondo, Y. Arimura, K. Nakabayashi, S. Ozaki, M. Yoshida, T. Yoshida, N. Tsusaka, T. Okazaki, S. Naoe, K. Uno, N. Tamura, Y. Yamanishi, A. Fukatsu, T. Oharaseki, and T. Nakayama, for comparative study of the diagnostic accuracy of ELISA systems and valuable discussions. This study was supported by grant No. SH44410 from the Japan Health Science Foundation and a grant for 'Research on Health Science focusing in Drug Innovation, International Collaborative Research' from the Ministry of Health, Labour and Welfare in Japan. We also thank colleagues Dr. David Jayne, Addenbrooke Hospital, Cambridge, UK, Richard A. Watts, School of Medicine, Health Policy and Practice, University of East Anglia, Norwich, UK, David G.I. Scott, Department of Rheumatology, Norfolk and Norwich University Hospital, Norwich, UK, and Dr. Niels Rasmussen, University of Copenhagen Health Sciences Hospital, and Dr. Vladimir Tesar for valuable discussions. 


\section{References}

-1 Takagi D, Nakamaru Y, Maguchi S, Furuta Y, Fukuda S: Otologic manifestations of Wegener's granulomatosis. Laryngoscope 2002;112:1684-1690.

$\checkmark 2$ Fujimoto S, Uezono S, Hisanaga S, Fukudome K, Kobayashi S, Suzuki K, et al: Incidence of ANCA-associated primary real vasculitis in the Miyazaki Prefecture: the first population based, retrospective, epidemiological survey in Japan. Clin J Am Soc Nephrol 2006;1:1016-1022.

3 Watts RA, Scott DGI, Jayne DRW, Suzuki K, Hashimoto H: A comparative study of the diagnostic accuracy of ELISA systems for the detection of anti-neutrophil cytoplasm antibodies available in Japan and Europe. Clin Exp Rheumatol 2008;26:1027-1033.

4 Watts RA, Gonzalez-Gay MA, Lane SE, Garcia-Porrua, Bentham G, Scott DG: Geoepidemilogy of systemic vasculitis: comparison of the incidence in two regions of Europe. Ann Rheum Dis 2001;60:170-172.

$\checkmark 5$ Kobayashi S, Yano T, Matsumoto Y, Numano F, Nakajima N, Yasuda K, Yutani C, Nakayama T, Tamakoshi A, Kawamura T, Ohno Y, Inaba Y, Hashimoto $\mathrm{H}$ : Clinical and epidemiologic analysis of giant cell (temporal) arteritis from a nationwide survey in 1998 in Japan: the first government-supported nationwide survey. Arthritis Rheum 2003;49: 594-598.

6 Watts RA, Scott DG, Jayne DR, Ito-Ihara T, Muso E, Fujimoto S, Harabuchi Y, Kobayashi S, Suzuki K, Hashimoto $\mathrm{H}$ : Renal vasculitis in Japan and the UK - are there differences in epidemiology and clinical phenotype? Nephrol Dial Transplant 2008;23:39283931.

7 Nakamaru Y, Maguchi S, Takizawa M, Fukuda S, Inuyama Y: The association between human leukocyte antigens (HLA) and cytoplasmic-antineutrophil cytoplasmic antibody (cANCA)-positive Wegener's granulomatosis in a Japanese population. Rhinology 1996;34:163-165.

-8 Seta N, Tajima M, Kobayashi S, Kawakami Y, Hashimoto H, Kuwana M: Autoreactive Tcell responses to myeloperoxidase in patients with antineutrophil cytoplasmic antibodyassociated vasculitis and in healthy individuals. Mod Rheumatol 2008;18:593-600.

$\checkmark 9$ Fujii A, Tomizawa K, Arimura Y, Nagasawa T, Y-Ohashi Y, Hiyama T, Mizuno S, Suzuki $\mathrm{K}$ : Epitope analysis of myeloperoxidase-specific anti-neutrophil cytoplasmic antibody (MPO-ANCA) associated glomerulonephritis. Clin Nephrol 2000;53:242-252.

-10 Arimura Y, Minoshima S, Kamiya Y, Tanaka U, Nakabayashi K, Kitamoto K, Nagasawa T, Sasaki T, Suzuki K: Serum myeloperoxidase and serum cytokines in anti-myeloperoxidase antibody-associated glomerulonephritis. Clin Nephrol 1993;40:256-264.
$>11$ Tomizawa K, Mine E, Fujii A, Ohashi Y, Yamagoe S, Ishida-Okawara A, Hashimoto Y, Ito M, Tanokura M, Yamamoto T, Arimura Y, Nagasawa T, Mizuno S, Suzuki K: A panel set for epitope analysis of myeloperoxidase (MPO)-specific anti-neutrophil cytoplasmic antibody MPO-ANCA using recombinant hexamer histidine-tagged MPO deletion mutants. J Clin Immunol 1998;18: 142-152.

12 Erdbrügger U, Hellmark T, Bunch DO, Alcorta1 DA, Jennette JC, Falk RJ, Nachman PH: Mapping of myeloperoxidase epitopes recognized by $\mathrm{MPO}$-ANCA using humanmice MPO chimeras. Kidney Int 2006;69: 1799-805.

13 van der Geld YM, Stegman CA, Kallenberg CGM: B cell epitope specificity in ANCAassociated vasculitis: does it matter? Clin Exp Immunol 2004;137:451-459.

14 Van der Geld YM, Huitema MG, Franssen CF, van der Zee R, Limburg PC, Kallenberg CGM: In vitro $\mathrm{T}$ lymphocyte responses to proteinase 3 (PR3) and linear peptides of PR3 in patients with Wegener's granulomatosis (WG). Clin Exp Immunol 2000;122: 504-513.

15 Ito-Ihara T, Muso E, Kobayashi S, Uno K, Tamura N, Yamanishi Y, Fukatsu A, Watts RA, Scott DGI, Jayne DRW, Suzuki K, Hashimoto H: A comparative study of the diagnostic accuracy of ELISA for the detection of anti-neutrophil cytoplasma antibodies available in Japan and Europe. Clin Exp Rheum 2008;26:1027-1033.

16 Nakamura Y, Yashiro M, Uehara R, et al: Epidemiologic features of Kawasaki disease in Japan: results of the 2007-2008 nationwide survey. J Epidemiol 2010;20:302-307.

17 Wu MH, Nakamura Y, Burns J, et al: Stateof-the-art basic and clinical science of Kawasaki disease. Pediatric Health 2008;2:405409.

18 Jennette JC, Falk RJ, Andrassy K, Bacon PA, Churg J, Gross WL, Hagen EC, Hoffman GS Hunder GG, Kallenberg CG, et al: Nomenclature of systemic vasculitides. Proposal of an international consensus conference. Arthritis Rheum 1994;37:187-192.

19 Weidner N: Giant-cell vasculitides. Semin Diagn Pathol 2001;18:24-33.

20 Lawrence RC, Helmick CG, Arnett FC, Deyo RA, Felson DT, Giannini EH, Heyse SP, Hirsch R, Hochberg MC, Hunder GG, Liang $\mathrm{MH}$, Pillemer SR, Steen VD, Wolfe F: Estimates of the prevalence of arthritis and selected musculoskeletal disorders in the United States. Arthritis Rheum 1998;41:778-799.
21 González-Gay MA, Alonso MD, Agüero JJ, Bal M, Fernández-Camblor B, Sánchez-Andrade A: Temporal arteritis in a northwestern area of Spain: study of 57 biopsy proven patients. J Rheumatol 1992;19:277-280.

22 Yamaguchi A, Ogawa A, Tsuchiya N, Shiota M, Mitsui H, Tokunaga K, Tadokoro K, Juji T, Ito K: HLA-B27 subtypes in Japanese with seronegative spondyloarthropathies and healthy controls. J Rheumatol 1996;23:11891193.

23 Numano F: Differences in clinical presentation and outcome in different countries for Takayasu's arteritis. Curr Opin Rheumatol 1997;9:12-15.

24 Chen M, Yu F, Zhang Y, Zou WZ, Zhao MH, Wang HY: Characteristics of Chinese patients with Wegener's granulomatosis with anti-myeloperoxidase autoantibodies. Kidney Int 2005;68:2225-2229.

$>25$ Kobayashi S, Yano T, Inaba Y, Hashimoto H, Matsumoto Y, Tamakoshi A, et al: Ocular involvements of Japanese patients with giant cell arteritis from the first nation-wide study. Arthritis Rheum 2003;49:867-868.

26 Tsuzuki K, Fukazawa K, Takebayashi H, Hashimoto K, Sakagami M: Difficulty of diagnosing Wegener's granulomatosis in the head and neck region. Auris Nasus Larynx 2009;36:64-70.

27 Hoffman GS, Kerr GS, Leavbitt RY, Hallahan CW, Lebovics RS, Travis W, et al: Wegener granulomatosis: an analysis of 158 patients. Ann Intern Med 1992;116:488-498.

-28 Tsuchiya N, Kobayashi S, Kawasaki A, Kyogoku C, Arimura Y, Yoshida M, Tokunaga K, Hashimoto H: Genetic background of Japanese patients with antineutrophily cytoplasmic antibody-associated vasculitis: association of HLA-DRB1*0901 with microscopic polyangiitis. J Rheumatol 2003;30:15341540

-29 Mamegano K, Kuroki K, Miyashita R, Kusaoi M, Kobayashi S, Matsuta K, Maenaka K, Colonna M, Ozaki S, Hashimoto H, Takasaki Y, Tokunaga K, Tsuchiya N: Association of LILRA2 (ILT1, LIR7) splice site polymorphism with systemic lupus erythematosus and microscopic polyangiitis. Genes Immun 2008;9:214-223.

>30 Miyashita R, Tsuchiya N, Yabe T, Kobayashi S, Hashimoto H, Ozaki S, Tokunaga K: Association of killer cell immunoglobulin-like receptor genotypes with microscopic polyangiitis. Arthritis Rheum 2006;54:992-997.

-31 Tsuchiya N, Kobayashi S, Hashimoto H, Ozaki S, Tokunaga K: Association of HLADRB1*0901-DQB1*0303 haplotype with microscopic polyangiitis in Japanese. Genes Immun 2006;7:81-84.

$>32$ Jayne DRW; European Vasculitis Study Group (EUVAS): Update on the European Vasculitis Study Group trials. Curr Opin Rheumatol 2001;13:48-55. 
>33 Koyama A, Yamagata K, Makino H, Arimura $Y$, Wada $T$, Nitta $K$, Nihei $H$, Muso $M$, Taguma Y, Shigematsu H, Sakai H, Tomino Y, Matsuo S, Japan RPGN Registry Group: A nationwide surveillance of rapidly progressive glomerulonephritis in Japan; etiology, prognosis and treatment diversity. Clin Exp Nephrol 2009; 13:633-650.

>34 Hogan SL, Nachman PH, Wilkman AS, JC Jennette JC, Falk RJ: Prognostic markers in patients with antineutrophil cytoplasmic autoantibody-associated microscopic polyangiitis and glomerulonephritis. J Am Soc Nephrol 1996;7:23-32.

>35 Hauer HA, Bajema IM, Van Houwelingen HC, Ferrario F, Noël LH, Waldherr R, Jayne DRW, Rasmussen N, Bruijn JA, Hagen EC, European Vasculitis Study Group (EUVAS): Renal histology in ANCA-associated vasculitis: differences between diagnostic and serologic subgroups. Kidney Int 2002;61:8089.

-36 Tidman M, Olamder R, Avalander C, Danielsson D: Patients hospitalized because of small vessel vasculitides with renal involvement in the period 1975-1955: organ involvement, ANCA patterns, seasonal attack rates and fluctuation of annual frequencies. J Intern Med 1988;244:133-141.

$\checkmark 37$ Booth AD, Almond MK, Burns A, Ellis P, Gaskin G, Neild GH, Plaisance M, Pusey CD, Jayne DRW: Outcome of ANCA-associated renal vasculitis: a 5-year retrospective study. Am J Kidney Dis 2003;41:776-784.

38 Matsumoto M, Kobayashi S, Hashimoto H, et al: Nationwide epidemiological survey of refractory vasculitis (anti-neutrophil cytoplasmic antibody (ANCA)-associated vasculitis, anti-phospholipid syndrome and temporal arteritis) in Japan. Annual report of the Research Committee on Intractable Vasculitides, the Ministry of Health and Welfare of Japan,1998, pp 15-23 (in Japanese).

39 Sakai K, Kurokawa K, Koyama A, et al: Committee for the Guidelines on Diagnosis and Therapy of Rapidly Progressive Glomerulonephritis: the guidelines for the management of rapidly progressive glomerulonephritis (in Japanese). Jpn J Nephrol 2002;44:55-83.

40 Usui J, Yamagata K: Present status and future perspective of RPGN in Japan (in Japanese). Jpn J Nephrol 2009;51:78-83.

-41 Lane SE, Scott DG, Heaton A, Watts RA: Primary renal vasculitis in Norfolk-increasing incidence or increasing recognition? Nephrol Dial Transplant 2000;15:23-27.

-42 Satchell SC, Nicholls AJ, D’Souza RJ, Beaman M: Renal vasculitis: increasing a disease of the elderly? Nephron Clin Prac 2004; 97:c142-c146.

-43 Uezono S, Sato Y, Hara S, Hisanaga S, Fukudome K, Fujimoto S, Nakao H, Kitamura K, Kobayashi S, Suzuki K, Hashimoto H, Nunoi $\mathrm{H}$ : Outcome of ANCA-associated primary renal vasculitis in Miyazaki Prefecture. Intern Med 2007;46:815-822.
44 Jayne D: Progress of treatment in ANCA-associated vasculitis. Nephrology 2009;14:4248

45 Suzuki K, Kobayashi S, Yamazaki K, Gondo M, Tomizawa K, Arimura Y, Nakabayashi K, Ozaki S, Yoshida M, Yoshida T, Tsusaka N, Muso E, Okazaki T, Hashimoto H: Analysis of risk epitopes of anti-neutrophil antibody MPO-ANCA in vasculitis in Japan population. Microbiol Immunol 2007;51:12151220.

46 Falk RJ, Jennette JC: ANCA are pathogenic - oh yes they are! J Am Soc Nephrol 2002; 13:1977-1979.

47 Heeringa ]P, Tervaert JWC: Pathophysiology of ANCA-associated vasculitides: are ANCA really pathogenic? Kidney Int 2004;65:15641567.

48 Suzuki R, Tomizawa K, Suzuki K, Tanokura $\mathrm{M}$ : MPO-ANCA Binding site on MPO molecule estimated from epitope mapping study and molecular modeling. Bioimages 2004 12:85-90.

49 Holle JU, Hellmich B, Backes M, Gross WL, Csernok E: Variations in performance characteristics of commercial enzyme immunoassay kits for detection of antineutrophil cytoplasmic antibodies: what is the optimal cut off? Ann Rheum Dis 2005;64:1773-1779.

50 Trevisin M, Pollock W, Dimech W, et al: Antigen-specific ANCA ELISAs have different sensitivities for active and treated vasculitis and for nonvasculitic disease. Am J Clin Pathol 2008;129:42-53.

51 Hagen EC, Andrassy K, Chernok E, et al: The value of indirect immunofluorescence and solid phase techniques for ANCA detection. A report on the first phase of an international cooperative study on the standardization of ANCA assays. EEC/BCR Group for ANCA Assay Standardization. J Immunol Methods 1993;159:1-16.

52 Pollock W, Dunster K, Rolland JM, Koh H, Savige J: A comparison of commercial and in-house ELISAs for antineutrophil cytoplasmic antibodies directed against proteinase 3 and myeloperoxidase. Pathology 1999; 31:38-43.

53 Kobayashi S, Ito A, Okuzaki D, Onda H, Yabuta N, Nagamori I, Suzuki K, Hashimoto $\mathrm{H}$, Nojima H: Expression profiling of PBMCbased diagnostic gene markers isolated from vasculitis patients. DNA Res 2008;15:253265.

54 Tougan T, Onda H, Okuzaki D, Kobayashi S, Hashimoto H, Nojima H: Focused microarray analysis of peripheral mononuclear blood cells from Churg-Strauss syndrome patients. DNA Res 2008;15:103-114.

55 Kinjoh K, Kyogoku M, Good RA: Genetic selection for crescent formation yields mouse strain with rapidly progressive glomerulonephritis and small vessel vasculitis. Proc Natl Acad Sci USA 1993;90:3413-3417.
56 Neumann I, Birck R, Newman M, Schnülle P, Kriz W, Nemoto K, Yard B, Waldherr R, Van Der Woude FJ: SCG/Kinjoh mice: a model of ANCA-associated crescentic glomerulonephritis with immune deposits. Kidney Int 2003;64:140-148.

57 Ishida-Okawara A, Ito-Ihara T, Muso E, Ono T, Saiga K, Nemoto K, Suzuki K: Neutrophil contribution to the crescentic glomerulonephritis in SCG/Kj mice. Nephrol Dial Transplant 2004;19:1708-1715.

58 Jethwa HS, Clarke SH, Itoh-Lindstrom Y, Falk RJ, Jennette JC, Nachman PH: Restriction in V kappa gene use and antigen selection in anti-myeloperoxidase response in mice. J Immunol 2000;165:3890-3897.

59 Hamano Y, Tsukamoto K, Abe M, Sun GD, Zhang D, Fujii H, Matsuoka S, Tanaka M, Ishida-Okawara A, Tachikawa H, Nishimura H, Tokunaka K, Hirose S, Suzuki K: Genetic dissection of vasculitis, myeloperoxidasespecific antineutrophil cytoplasmic autoantibody production, and related traits in spontaneous crescentic glomerulonephritisforming/Kinjoh mice. J Immunol 2006;176: 3662-3673.

60 Kawasaki T: Acute febrile mucocutaneous syndrome with lymphoid involvement with specific desquamation of fingers and toes in children. Clinical observation of 50 patients (in Japanese). Jpn J Allergy 1967;16:178-222.

-61 Kawasaki T, Kosaki F, Okawa S, et al: A new infantile acute febrile mucocutaneous lymph-node syndrome (MLNS) prevailing in Japan. Pediatrics 1974;54:271-276.

62 Ayusawa M, Sonobe T, Uemura S, et al, Kawasaki Disease Research Committee: Revision of diagnostic guidelines for Kawasaki disease (the 5 th revised edition). Pediatr Int 2005;47:232-234.

63 Masuda H, Naoe S, Tanaka N: A pathological study of coronary artery in Kawasaki disease (MCLS) - with special reference to morphogenesis of aneurysm (in Japanese). J Jpn Coll Angiol 1981;21:899-912.

64 Landing HL, Larson E: Pathological features of Kawasaki disease (mucocutaneous lymph node syndrome). Am J Cardiovasc Pathol 1987;1:215-229.

65 Takahashi K, Oharaseki T, Naoe S, et al: Neutrophilic involvement in the damage to coronary arteries in acute stage of Kawasaki disease. Pediatr Int 2005;47:305-310.

66 Takahashi K, Oharaseki T, Naoe S: Pathological study of postcoronary arteritis in adolescents and young adults, with reference to the relationship between sequelae of Kawasaki disease and atherosclerosis. Pediatr Cardiol 2001;22:138-142.

67 Furusho K, Kamiya T, Nakano H, et al: Highdose intravenous gamma globulin for Kawasaki disease. Lancet 1984;2:1055-1058.

68 Newburger JW, Takahashi M, Burns JC, et al: The treatment of Kawasaki syndrome with intravenous gamma globulin. N Engl J Med 1986;315:341-347. 
-69 Newburger JW, Sleeper LA, McCrindle BW, et al, Pediatric Heart Network Investigators: Randomized trial of pulsed corticosteroid therapy for primary treatment of Kawasaki disease. N Engl J Med 2007;356:663-675.

-70 Inoue Y, Okada Y, Shinohara M, et al: A multicenter prospective randomized trial of corticosteroids in primary therapy for Kawasaki disease: clinical course and coronary artery outcome. J Pediatr 2006;149:336-341.

71 Nakano M, Yamada S. Hayashi Y, et al: Preventive effects of ulinastatin for coronary artery aneurysm formation in Kawasaki Disease; in Kato H (ed): Kawasaki Disease. Amsterdam, Elsevier Science, 1995, pp 364371.

72 Mori M, Imagawa T, Katakura S, et al: Efficacy of plasma exchange therapy for Kawasaki disease intractable to intravenous gamma-globulin. Mod Rheumatol 2004;14: 43-47.
3 Burns JC, Mason WH, Hauger SB, et al: Infliximab treatment for refractory Kawasaki syndrome. J Pediatr 2005;146:662-667.

74 Murata H, Naoe S: Experimental Candidainduced arteritis in mice - relation to arteritis in Kawasaki disease. Prog Clin Biol Res 1987;250:523.

75 Lehman TJ, Warren R, Gietl D, et al: Variable expression of Lactobacillus casei cell wallinduced coronary arteritis: an animal model of Kawasaki's disease in selected inbred mouse strains. Clin Immunol Immunopathol 1988;48:108-118.

76 Philip S, Lee WC, Liu SK, et al: A swine model of horse serum-induced coronary vasculitis: an implication for Kawasaki disease. Pediatr Res 2004;55:211-219.
77 Oharaseki T, Kameoka Y, Kura F, Persad AS, Suzuki K, Naoe S: Susceptibility loci to coronary arteritis in animal model of Kawasaki disease induced with Candida albicans-derived substances. Microbiol Immunol 2005; 49:181-189.

78 Nagai-Miura N, Shingo Y, Adachi Y, IshidaOkawara A, Oharaseki T, Takahashi K, Naoe S, Suzuki K, Ohno N: Induction of coronary arteritis with administration of CAWS (Candida albicans water-soluble fraction) depending on mouse strains. Immunopharmacol Immunotoxicol 2004;26:527-543.

79 Takahashi K, Oharaseki T, Yokouchi Y, Miura NN, Ohno N, Okawara AI, Murata H, Naoe S, Suzuki K: Administration of human immunoglobulin suppresses development of murine systemic vasculitis induced with Candida albicans water-soluble fraction: an animal model of Kawasaki disease. Mod Rheumatol 2010;20:160-167. 